\title{
Choosing Wisely Canada seeks system change
}

$\mathrm{E}$ ducation and awareness won't be enough to change the culture of waste in medicine, said speakers and participants at the inaugural Choosing Wisely Canada meeting held in Toronto. Now heading into its third year, the campaign is shifting gears from raising awareness of potentially unnecessary tests and treatments to helping health care workers change systems and habits that promote waste.

"In Canada, we deliver good care in spite of the system, not because of the system," keynote speaker and veteran health reporter André Picard told attendees at the Mar. 30 meeting. "Structural change is the area where we most need to wise up."

Choosing Wisely Canada's early strategy of publishing lists of procedures that doctors and patients should question has been successful as a conversation starter, "but we have to be careful not to let our enthusiasm get the better of us," Picard said.

He cited a JAMA Internal Medicine study on the impact of the American Choosing Wisely campaign as a "cautionary tale" of what happens when education isn't paired with structural change. The study showed a modest decrease in insurance claims for only two out of seven low-value services after the release of Choosing Wisely recommendations, the clinical significance of which was "uncertain."

"The study proved that if you do nothing, you get no results," Picard explained. "It's not enough to publish the lists; you have to act on them and push them relentlessly."

To that end, Choosing Wisely Canada debuted five toolkits at the meeting to help health workers jumpstart projects in their organizations. The toolkits are based on initiatives that have successfully reduced unnecessary procedures, and include templates for revising existing processes to support the campaign's recommendations.

"A lot of waste has been baked into

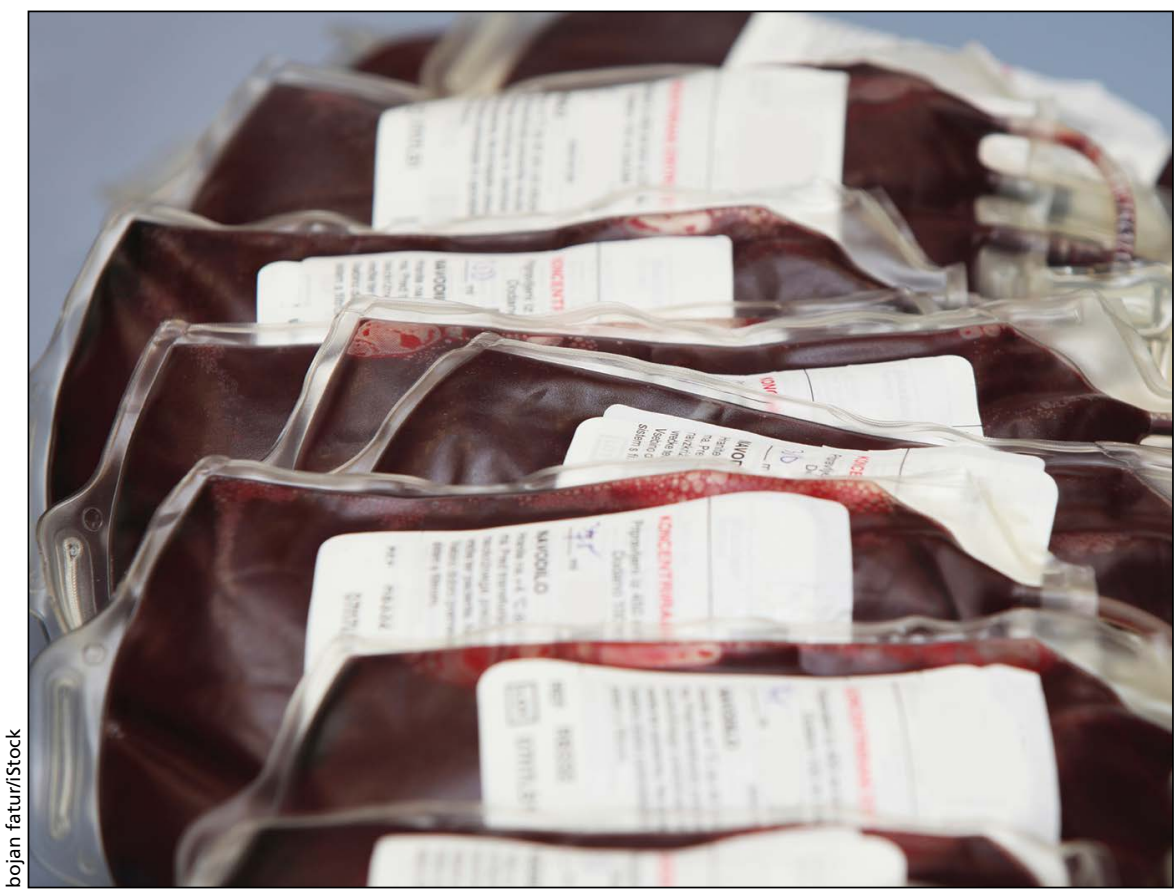

New Choosing Wisely Canada toolkits tackle systemic waste, including unnecessary blood transfusions.

our systems, into our order sets, into our medical directives," says Tai Huynh, Choosing Wisely Canada's campaign manager. "We want to encourage a culture of local ingenuity."

Some 70 projects were showcased at the meeting. The most successful tend to blend "low leverage" interventions like awareness campaigns with "high leverage" interventions like adding Choosing Wisely recommendations into electronic medical records and decision-support tools, says Huynh. "Somewhere in between are things like performance measurement and audit feedback."

North York General Hospital in Toronto, for example, cut emergency department lab testing by $31 \%$ since September 2014, preoperative clinic lab testing by $38 \%$ since February 2015 and intensive care unit mobile chest $\mathrm{x}$-rays by $12 \%$ since January 2015. The hospital achieved these reductions by integrating recommendations into computerized physician order entry systems, order sets and medical directives, as well as using staff screensavers, intranet, blogs and social media to share the changes with health workers and patients.

"It's about exposure, exposure, exposure," says Dr. Donna McRitchie, vice president of medical and academic affairs at North York General Hospital. "It was constantly revisiting and reeducating, showing what great work we had done and using it as a springboard for other things." Having the recommendations built into electronic records ensures that exposure is automatic, she adds.

Nova Scotia's former Capital District Health Authority took a more topdown approach to reducing unnecessary blood transfusions. "We came up with a policy and we enforced the policy: the blood transfusion service will not issue a second unit of blood cells until you reassess the patient and tell us why you need the second unit," explains Dr. Irene Sadek, medical director of pathology and laboratory medicine at Queen Elizabeth II Health Sciences Centre in 
Halifax. "Ninety percent of the time if the patient is not bleeding and is stable one unit will be enough."

This gatekeeper policy, which excluded emergency and outpatient departments, resulted in a $16.4 \%$ decline in red blood cell transfusions. "Having a policy might only help one or two percent of people who are keen on following it; education could give you maybe a $5 \%$ reduction, but a few months later people forget about it. What's most helpful is enforcement," Sadek says.

Other facilities, including St. Michael's Hospital in Toronto, have helped frontline staff develop and enforce their own interventions. "Our hypothesis was that if we provided the infrastructure support that frontline workers needed to engage in this issue, they would do it," says Dr. Lisa
Hicks, Choosing Wisely lead at St. Michael's. "So far that's been true."

"What I found has been really essential has been getting teams through some of the bureaucratic hurdles that are present in the beginning of projects, helping them get the necessary approvals, and connecting them with the right people to get things done," she adds. "Hospitals are large bureaucracies and that's where we can help."

This approach has allowed St. Michael's to run 10 different Choosing Wisely initiatives simultaneously, which in turn has increased awareness of the campaign, Hicks says. It's still too early to determine the success of most of these hyper-local interventions. However, one of the longest running projects has reduced testing for thrombophilia by about $50 \%$.

"For the inpatient environment we restricted access to the test and used our computer physician order entry system to provide an alert that approval was necessary," Hicks explains. For outpatient clinics, which don't have computerized order entry systems, the hospital allowed unrestricted access to those with expertise in thrombophilia testing, and required other clinics to refer to those experts.

"This is going to be a long journey and something that we have to work at, probably indefinitely. We want to build this into how we think about medicine," says Hicks. "Education alone is not an implementation strategy. If that's all that we do, I don't think we should expect things to change very much." - Lauren Vogel, CMAJ

CMAJ 2016. DOI:10.1503/cmaj.109-5262 